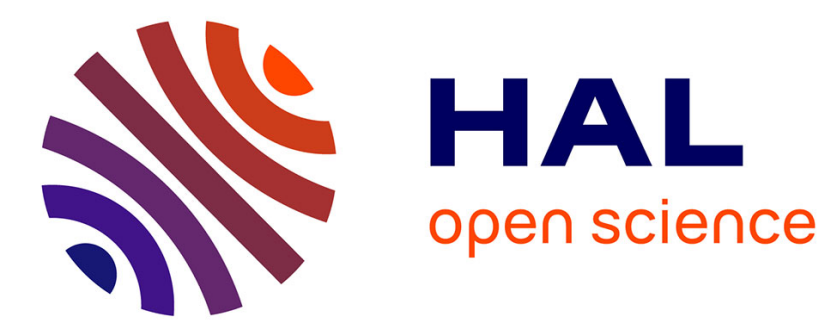

\title{
Study of $\mathrm{Hg}^{*}$ (63 p1)-noble gas collisions in a $200 \mathrm{kG}$ field range
}

J.C. Gay, A. Omont

\section{To cite this version:}

J.C. Gay, A. Omont. Study of Hg* (63 p1)-noble gas collisions in a $200 \mathrm{kG}$ field range. Journal de Physique Lettres, 1976, 37 (4), pp.69-73. 10.1051/jphyslet:0197600370406900 jpa-00231240

\section{HAL Id: jpa-00231240 https://hal.science/jpa-00231240}

Submitted on 1 Jan 1976

HAL is a multi-disciplinary open access archive for the deposit and dissemination of scientific research documents, whether they are published or not. The documents may come from teaching and research institutions in France or abroad, or from public or private research centers.
L'archive ouverte pluridisciplinaire HAL, est destinée au dépôt et à la diffusion de documents scientifiques de niveau recherche, publiés ou non, émanant des établissements d'enseignement et de recherche français ou étrangers, des laboratoires publics ou privés. 


\title{
STUDY OF $\mathrm{Hg}^{*}\left(6^{3} \mathrm{P}_{1}\right)$-NOBLE GAS COLLISIONS IN A 200 kG FIELD RANGE
}

\author{
J. C. GAY and A. OMONT \\ Laboratoire de Spectroscopie Hertzienne de l'E.N.S. (*) \\ et Université Paris VII \\ 4, place Jussieu, Tour 12, E01, 75230 Paris Cedex 05, France
}

(Reçu le 19 décembre 1975, accepté le 22 janvier 1976)

\begin{abstract}
Résumé. - On donne les résultats de l'étude des collisions $\mathrm{Hg}^{*}\left(6{ }^{3} \mathrm{P}_{1}\right)$-gaz rares dans un domaine de champ magnétique de $200 \mathrm{kG}$. Les résultats obtenus montrent que les taux de transfert par collisions entre les sous-niveaux $m=0$ et $m= \pm 1$ d'un isotope pair du mercure ont un comportement extrêmement différent en fonction du champ selon le gaz rare perturbateur considéré. L'interprétation qualitative des résultats est ébauchée à l'aide d'un modèle théorique prévoyant une forte anisotropie de la relaxation en présence du champ magnétique.

Abstract. - The study of $\mathrm{Hg}^{*}\left(6{ }^{3} \mathrm{P}_{1}\right)$-rare gas collisions in a $200 \mathrm{kG}$ range of magnetic field is reported. The results show that the collisional transfer rates between the $m=0$ and the $m= \pm 1$ Zeeman sublevels of an even isotope of mercury exhibit a strikingly different dependence on the field strength according to the noble gas species. A very qualitative attempt at interpretation is given using the results of a previous theoretical model which predicts a strong anisotropy of the relaxation process when the vapour is submitted to such magnetic fields.
\end{abstract}

1. Introduction. - We have recently reported the results of a study of the magnetic field dependence of the collisional transfer rates between Zeeman sublevels in $\mathrm{Hg}-\mathrm{Hg} *\left({ }^{3} \mathrm{P}_{1}\right)$ resonant collisions and $\mathrm{Hg}^{*}\left(6^{3} \mathrm{P}_{1}\right)$-rare gas non-resonant collisions in a $80 \mathrm{kG}$ field range [1]. We now present the results of a study on $\mathrm{Hg}^{*}$-rare gas collisions in a wider $(200 \mathrm{kG})$ range of the field.

As a recent theoretical investigation has shown [2], [3], a very strong magnetic field causes a variation of the collisional transfer rates between the Zeeman sublevels and is also responsible for a very important anisotropy in the relaxation process. The anisotropy is revealed for example by the fact that the transfer rates between the $m=0$ and $m= \pm 1$ Zeeman sublevels of the excited state are different. The behaviour with the field of the transfer rates is connected with the sign of the anisotropy of the interaction. Consequently we have recorded in these new experimental investigations both the ratio of the fluorescence intensities $I_{\sigma} / I_{\pi}$ emitted in the direction perpendicular to the field and the ratio $I_{\sigma}-/ I_{\sigma^{+}}$of the circularly polarized components of the light emitted in the direction of the field.

2. Experimental. - The arrangement we used is similar to that described in a previous paper [1]. Instead of the $80 \mathrm{kG}$ superconductive magnet of the

$\left(^{*}\right)$ Associé au C.N.R.S., LA, ${ }^{\circ} 18$.
Laboratory, we use the 130 and $190 \mathrm{kG}$ Bitter coils of the Service National des Champs Intenses (C.N.R.S. Grenoble).

The experiments are performed on the $6{ }^{3} \mathrm{P}_{1}$ state of ${ }^{198} \mathrm{Hg}$ (purity better than $99.7 \%$ ). The resonance cells filled with this isotope and containing one of the various noble gases are placed at the center of the Bitter coils. A ${ }^{198} \mathrm{Hg}$ R.F. discharge lamp in zero field is used to excite the $m=0$ Zeeman sublevel. This level is shifted by the field because of a slight decoupling of the fine structure. The value of the shift is $0.8 \mathrm{GHz}$ at $200 \mathrm{kG}$. This shift is smaller than the width of the line emitted by the lamp and does not cause any difficulty in the experiments.

We detect both the $\sigma$ and $\pi$ fluorescence intensities emitted at right angles to the excitation direction and the $\sigma^{+} \sigma^{-}$circularly polarized intensities emitted in the direction of the field (Fig. 1). The strongly shielded

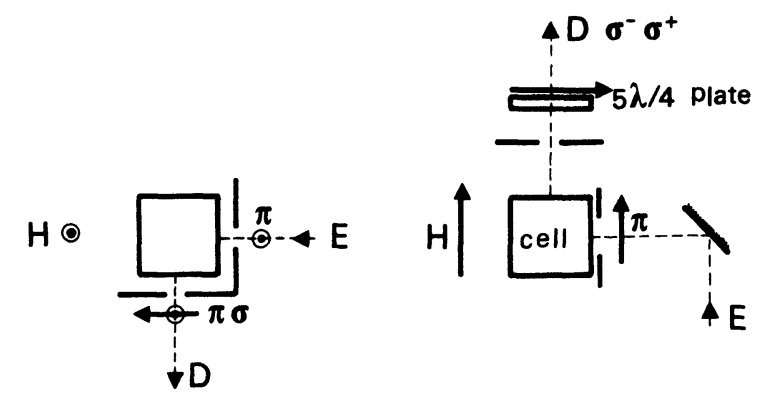

Fig. 1. - Geometrical arrangement of the beams for the two directions of detection. 
photomultiplier and lamp are placed at about 4 meters from the magnet. Optical excitation and detection are achieved by using flexible or rigid fused silica light guides.

We can insert various mercury absorption filters filled with pure isotopes into the detection beam. A ${ }^{198} \mathrm{Hg}$ filter is used to absorb the small amount of $\pi$ light which is mixed with the $\sigma$ light because of the imperfections of the detection polarizer, or which is mixed with the $\sigma^{+}, \sigma^{-}$circular fluorescence light because of the non zero aperture of the detection beam.

We have verified that there are no stray field effects on the PM or on the lamp. The shield of the PM is designed for 50 gauss which is three times the maximum value of the stray field of the $200 \mathrm{kG}$ Bitter coil.

3. Theoretical interpretation of the signals. - We assume that the transfer rates between Zeeman sublevels are $a$ priori different which is clearly evident when the field is non zero. The stationary solution of the master equation for the populations $\left(\rho_{m}\right)$ of the $m$ Zeeman sublevels is given by :

$$
\begin{aligned}
\dot{\rho}_{m}=0=-\left(\Gamma^{m}+\sum_{p \neq m} g^{p m}\right) & \rho_{m}+ \\
& +\sum_{p \neq m} g^{m p} \rho_{p}+\lambda_{0} \delta_{m 0}
\end{aligned}
$$

where $g^{p m}$ represents the transfer rate from the $m$ to the $p$ Zeeman sublevel, $\Gamma^{m}$ is the inverse of the imprisonment time of the photons emitted by the $m$ sublevel and $\lambda_{0} \delta_{m 0}$ is the pumping term describing the excitation of the $m=0$ level.

3.1 LOW MERCURY DENSITY EXPERIMENTAL CONDITIONS. - In order to simplify the interpretation of the experimental results, we have chosen conditions such that :

- $\Gamma^{m} \simeq \Gamma$, i.e. weak mercury densities in the cell which can be achieved by maintaining the side arm of the cell at low temperatures $\left(T \simeq-34^{\circ} \mathrm{C}\right)$. In these conditions, multiple scattering of the resonance photons is almost negligible. We thus avoid some of the problems connected with the lack of homogeneity of the magnet [1]. Besides, as self absorption is nearly negligible, the intensities issuing from the cell are nearly proportional to the populations of the Zeeman sublevels.

- $\Gamma \gg \sum_{p \neq m} g^{p m}$ which means that the backward terms due to collisions are negligible during the lifetime of the excited state. This condition is fulfilled by choosing the pressure of the rare gas contained in the cell. We used cells containing respectively 0.7 torr $\mathrm{Ne}, 0.3$ torr $\mathrm{Xe}, 0.5$ torr $\mathrm{Kr}, 0.6$ torr $\mathrm{A}, 0.6$ torr $\mathrm{He}$. This gives values of $g^{10} / \Gamma$ of about 0.2 which insures that the fluorescence intensities are almost proportional to the collisional rates.
Under these conditions, the expressions for the intensities are quite simple.

One obtains :

$$
\begin{aligned}
& R_{\mathrm{C}}=\frac{I_{\sigma^{-}}}{I_{\sigma^{+}}}=\frac{\rho_{-1}}{\rho_{+1}} \simeq \frac{g^{-10}}{g^{10}} \\
& R_{\mathrm{L}}=\frac{I_{\sigma}}{I_{\pi}}=\frac{\rho_{-1}+\rho_{+1}}{2 \rho_{0}} \simeq \frac{g^{-10}+g^{10}}{2 \Gamma} .
\end{aligned}
$$

3. 2 High MERCURY DENSITY LIMIT. - On the other hand, when the temperature of the side arm of the cell is high $\left(T \simeq 20^{\circ} \mathrm{C}\right.$ - mercury density about $\left.10^{13} \mathrm{at} / \mathrm{cm}^{3}\right)$, the imprisonment time in the vapour is about twenty times the natural lifetime. Then $g / \Gamma$ is about 4 . In these conditions the backward terms are very important, and thermal equilibrium is nearly established in the cell. The ratio of the \pm 1 populations is then given by the Boltzmann law

$$
I_{\sigma^{-}} / I_{\sigma^{+}}=\rho_{-1} / \rho_{+1} \simeq \mathrm{e}^{2 \hbar \omega / k T} .
$$

One could reach the same equilibrium at low mercury densities by choosing higher rare gas pressures, e.g. values of $g^{10} / \Gamma$ of about 4 .

4. Results. - We successively give the results for linear $(\sigma / \pi)$ and circular $\left(\sigma^{-} / \sigma^{+}\right)$polarizations.

4.1 LinEAR POLARIZATION. - The values of $I_{\sigma} / I_{\pi}$ normalized at the values in low field $(H \simeq 10 \mathrm{kG})$ and corrected for the imperfections ( $3 \%$ ) of the polarizer are given in figure 2 as a function of the field, for the various noble gas at low mercury densities

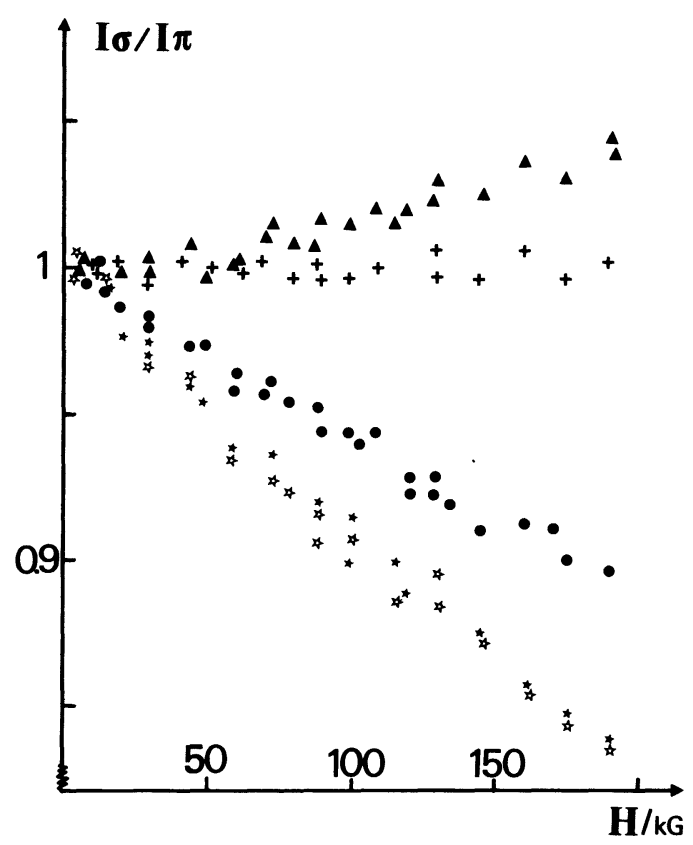

FIG. 2. - Variations with the field $H$ of $I_{\sigma} / I_{\pi}$ (normalized at the values for $H \simeq 10 \mathrm{kG}$ ) for the vasious rare gases. The temperature of the side arm of the cell is $T \simeq-34^{\circ} \mathrm{C}: \checkmark \nsucc$ xenon (two different cells), $\bullet$ krypton, + argon, $\Delta$ neon. 
$\left(<10^{11} \mathrm{at} / \mathrm{cm}^{3}\right)$. The curves are not given in the case of helium. (The variations are $0 \pm 3 \%$ in this case with a poor signal to noise ratio.)

The results are uncorrected for the effects of the spurious light of the lamp (the light which is scattered or reflected from the walls of the cell without undergoing absorption or emission in the vapour). This correction was very difficult to make in our experimental conditions. It could introduce a systematic error in the curves of figure 2 lessening the total variation with the field by no more than $3 \%$.

Each curve represents the average of several runs under similar conditions. In the case of xenon we used two different cells containing nearly the same xenon pressure.

The variations with the field in the cases at $60 \mathrm{kG}$ of $\mathrm{Kr}$ and $\mathrm{Xe}$ seem to be less pronounced but not inconsistent with those obtained in reference [1], when the effect of backwards terms $( \pm 1 \rightarrow 0)$ on the determination of the value of $\left(g^{-10}+g^{10}\right) / 2 \Gamma$ is taken into account.

We can compare the variations qualitatively with the field of $\left(g^{-10}+g^{10}\right)$ with those predicted in the theoretical model of reference [2] assuming a $R^{-6}$ Van der Waals potential. The experimental variations of about $20 \%$ and $12 \%$ respectively obtained for Xe and $\mathrm{Kr}$ are smaller than those predicted with the theoretical model using the measurements in zero field [4], [5] to fit the strength of the potential. This may be due to the necessity to introduce a repulsive part in the model potential which has not been done in the theoretical model of reference [2].

There is no significant variation with the field in the case of argon which is not in agreement with a $R^{-6}$ attractive Van der Waals potential.

Unexpected and needing to be confirmed is the behaviour with the field in the case of neon. In this case it is of course inappropriate either to compare to a $R^{-6}$ potential or to admit that the other hypothesis of the theoretical model are valid.

4.2 Circular polarizations. - In figure 3, we report the values of the ratio $R_{\mathrm{C}}=I_{\sigma^{-}} / I_{\sigma^{+}}$deduced from experimental measurements for the various noble gas at low mercury vapour pressure. $I_{\sigma^{+}}$always denotes the intensity of the light emitted by the $m=+1$ Zeeman sublevel. The values of the intensities are corrected for the imperfections of the circular analyzer ( $5 \lambda / 4$ wave plate and polarizer). The imperfection is evaluated by scanning the absorption of a ${ }^{199} \mathrm{Hg}$ cell interposed on the detection beam when varying the field on the main ${ }^{198} \mathrm{Hg}$ cell. Absorption signals occur when the frequency of a $\sigma$ component of ${ }^{198} \mathrm{Hg}$ coincides with the frequency of a zero field hyperfine component of ${ }^{199} \mathrm{Hg}$. This gives both the values of the transmittances of the circular analyzer for the two $\sigma^{+}$and $\sigma^{-}$polarizations and an estimation of the spurious light from the lamp and of the $\pi$ light transmitted in spite of the ${ }^{198} \mathrm{Hg}$ filter

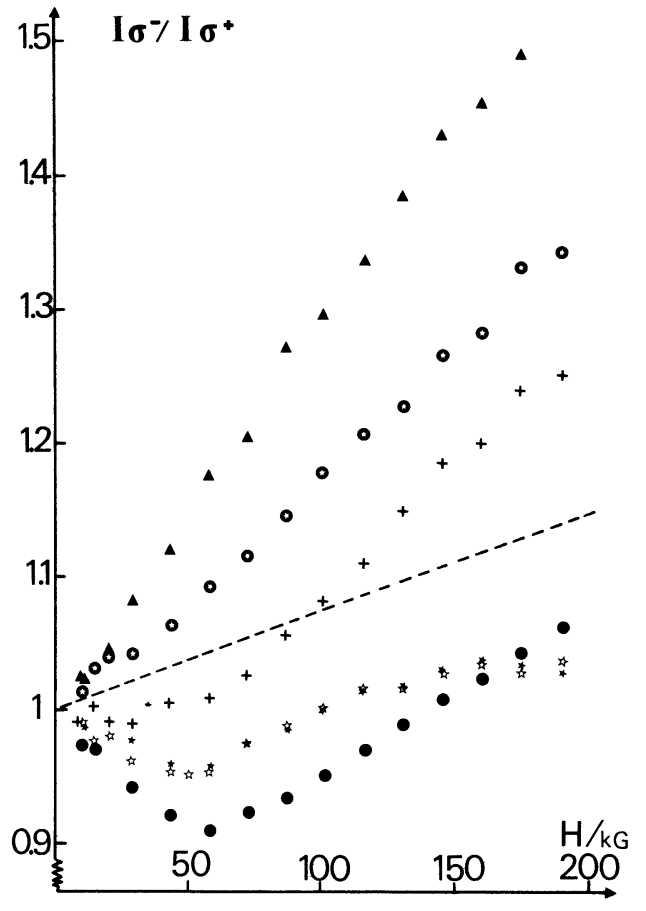

Fig. 3. - Variations with the field at $T \simeq-34^{\circ} \mathrm{C}$ of the ratio $I_{\sigma^{-}} / I_{\sigma^{+}}$. In these conditions $I_{\sigma^{-}} / I_{\sigma^{+}}$almost equals $g^{-10} / g^{10}$ : $¥$ \& xenon, $\bullet$ krypton, + argon, $\Delta$ neon, $\bullet$ helium. The dashed curve corresponds to $\mathrm{e}^{2 \hbar \omega / \boldsymbol{k} T}$.

in zero field placed in the detection beam. The imperfection of the analyzer is found to be about $7 \%$, the contribution of the spurious light of the lamp is also some percent of the $\sigma^{+}, \sigma^{-}$signals at low mercury densities.

The ratios $I_{\sigma^{-}} / I_{\sigma^{+}}$at low mercury densities differ by a few percent for the two opposite directions (up and down) of the field. The difference between the ratios seems to decrease in very high fields. Moreover, the values of the ratios obtained at high mercury densities for the two opposite directions of the field tend to coincide and do not exhibit any abnormal behaviour with the field. Although the origin of this phenomena is not clearly understood, it may probably be attributed to the polarization of the spurious light from the lamp. At low mercury densities, this spurious light is not quite negligible; but it is at high mercury densities. The dependence of the difference of the ratios on the field might be attributed to some magnetorotation effects on the polarization of the spurious light. (The magnetorotation produced by the $1 \mathrm{~mm}$ thick wall of the fused silica cell is $36^{\circ}$ at $200 \mathrm{kG}$.) We stress the fact that the qualitative behaviour of the ratio with the field is not modified by this parasitic effect. We have assumed that the above mentioned effects are nearly cancelled by just taking the average of the two values of $I_{\sigma^{-}} / I_{\sigma^{+}}$obtained for the two opposite directions of $\mathbf{H}$ (the axis of quantization is always taken along $\mathbf{H}$ ).

Although it is hard to be sure that there is no 
contribution in the observed variations of $I_{\sigma^{-}} / I_{\sigma^{+}}$ with $\mathbf{H}$ arising from magnetorotation or other similar magneto effects, it seems highly improbable that such effects are very important because the values of $R_{\mathrm{C}}$ measured in the case of high mercury vapour pressures exactly coincide with the values at local thermal equilibrium as expected (Fig. 4).

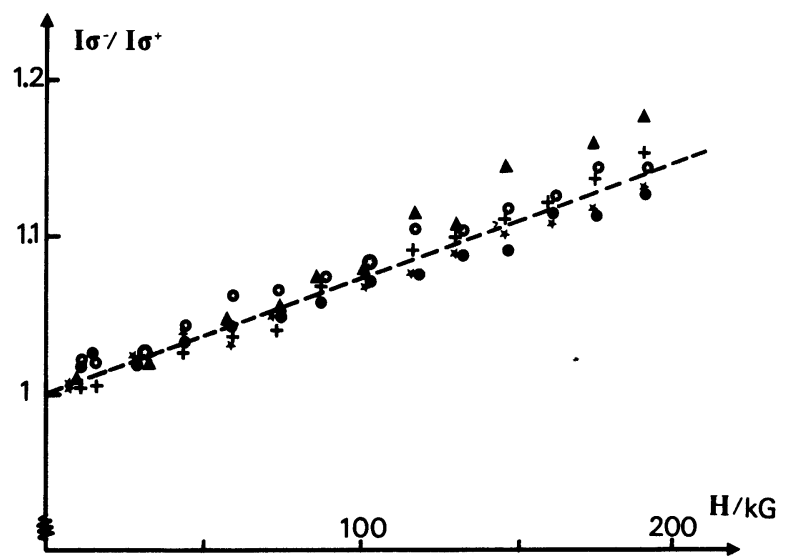

FIG. 4. - Variations with the field at room temperature of the ratio $I_{\sigma}-/ I_{\sigma^{+}}$for the various rare gases. The ratio in this case is nearly $\mathrm{e}^{2 \hbar \omega / k T}$ (dashed curve).

The results presented on figure 3 obtained at low mercury densities $\left(T=-34^{\circ} \mathrm{C}\right)$ have been corrected in the way discussed above. They were obtained with the $10 \mathrm{MW}$ vertical coil of S.N.C.I. Similar results were obtained in the range $0-130 \mathrm{kG}$ with a $5 \mathrm{MW}$ coil. Results obtained below $10 \mathrm{kG}$ are discarded because of possible coincidences with the frequency of the light emitted by isotopic impurities of the lamp.

As seen, the field variations of $I_{\sigma^{-}} / I_{\sigma^{+}}$are very large and quite different following the foreign gas under consideration. It should be noted that the results obtained with two different cells filled with xenon at comparable pressures ( $\sim 0.3$ torr) exactly coincide and that the depth observed in the case of xenon is no more than 0.02 at $T=-12^{\circ} \mathrm{C}$.

The results definitely show that $g^{-10} / g^{10}$ (proportional to $I_{\sigma^{-}} / I_{\sigma^{+}}$if backwards transition are negligible $\left({ }^{1}\right)$, cf. eq. (1)) is generally very different from 1 .

At mercury pressures corresponding to room temperature the imprisonment time is very large $\left(\sim 20 \mathrm{~T}^{-1}\right)$. Several collisions then occur before the escape of radiation. So the populations of the Zeeman sublevels are nearly distributed according to thermal equilibrium whatever the ratios of $g^{10}, g^{-10}$ and $g^{-11}$ are. This is confirmed by the experimental results plotted on figure 4 , where it is seen that $I_{\sigma^{-}} / I_{\sigma^{+}}$nearly equals $\mathrm{e}^{2 \hbar \omega / k T}$. It can be shown that taking into account the finiteness of the imprisonment time reduces the

( $\left.{ }^{1}\right)$ Taking into account these backwards transitions would modify the value of $g^{-10} / g^{10}$ by a few percent. difference between the observed values of $I_{\sigma^{-}} / I_{\sigma^{+}}$ and the theoretical value slightly different from $\mathrm{e}^{2 \hbar \omega / k T}$ to less than 0.02 for all foreign gases.

5. Interpretation. - A detailed interpretation of the observed variations of $g^{10}$ and $g^{-10}$ with respect to the field is very difficult because it would require a precise knowledge of $\mathrm{Hg}\left(6{ }^{3} \mathrm{P}_{1}\right)$-noble gas potential curves. We will therefore limit our comments to a few qualitative ones based on the theoretical model [2] we have developed:

- For krypton and xenon, $I_{\sigma^{-}} / I_{\sigma^{+}}$presents a minimum smaller than 1 at intermediate magnetic field $(\simeq 50 \mathrm{kG})$. This is in agreement with the theoretical predictions [2] for a $R^{-6}$ dispersion potential with a magnitude in accordance with the relaxation rates measured in zero-field and an anisotropy such that the energy of the $\Sigma$ level is greater than the energy of the $\Pi$ levels [4]. This agrees with the wellknown fact that the $R^{-6}$ long range potential is dominant for interactions with heavy and very polarizable noble gases $(\mathrm{Kr}$ and $\mathrm{Xe})$ [3]. The depth of the minimum is smaller than predicted by the theory $(0.72$ and 0.85 with velocity averaging), however this fact seems to be easily accounted for by different aspects such as very short range collisions, effects of the repulsive part of the potential, deviations from linear path which are not taken into account in the theoretical model.

- For light noble gases ( $\mathrm{He}, \mathrm{Ne}$ ), the repulsive part of the potential is well known to be the most important. The repulsive part of the anisotropy is likely to be such that the $\Sigma$ level is below the $\Pi$ levels [4]. Thus, it is not surprising that $I_{\sigma}-/ I_{\sigma^{+}}$is greater than one. If a maximum was to be expected by analogy with the minimum predicted for the $R^{-6}$ attractive potential, it would be located at higher field values because of the shorter range of the interaction and the much larger relative velocity. But there is no reason to be sure that a maximum does exist in this case. The faster increase observed with neon compared to helium in the field range of our experiments seems also to be explained by its smaller velocity.

- The case of argon is known to be intermediate. The attractive part of the potential is probably not negligible and could possibly explain the stationary behaviour observed up to $60 \mathrm{kG}$. However the effects of the repulsive part are probably important and would explain the fast increase of $I_{\sigma^{-}} / I_{\sigma^{+}}$observed at higher fields.

Let us stress that the results given in reference [2] suppose that the energy of the $\Sigma$ state is lower than the energy of the $\Pi$ states and that the inverse situation is obtained by only interchanging the $m=+1$ and $m=-1$ Zeeman sublevels in the results. Moreover, the conclusions are qualitatively unaltered whatever the $R^{-n}$ dependence of the potential. 
6. Conclusion. - All these explanations are tentative and need to be confirmed by a detailed study of the collision dynamics with different model potentials and by carefully checking the influence of the various approximations (straight line in particular). Such a study is currently in progress.

Let us also insist upon the necessity of new experiments to confirm and clarify the present results. It would be highly desirable to proceed to different straightforward extensions such as higher fields, higher temperatures, other foreign gases, etc., as well as to the study of other levels and other elements. In the light of the present results, such studies appear very promising and important to the knowledge of interatomic potentials of excited states. However, a parallel development of the theory of the dynamics of such collisions is clearly required in order to quantitatively relate the important observed variations of the collision rates to the interatomic potentials.

Acknowledgements. - We thank our colleagues of S.N.C.I. for helpful assistance during this work.

\section{References}

[1] GaY, J. C. and SchneIDer, W. B., J. Physique Lett. 36 (1975) L-185.

see also Fuchs, B., Hanle, W., Oberheim, W. and ScharmanN, A., Phys. Lett. 50 (1974) 337.

[2] GAY, J. C., J. Physique Lett. 36 (1975) L-239.
[3] Gay, J. C., soumis à J. Physique.

[4] Faroux, J. P., Thèse d'Etat, Paris (1969).

[5] Barrat, J. P., Casalta, D., Cojan, J. L. et Hamel, J., J. Physique 27 (1966) 608. 